\title{
A GENERAL FIXED POINT THEOREM FOR IMPLICIT CYCLIC MULTI-VALUED CONTRACTION MAPPINGS
}

\author{
VALERIU POPA
}

\begin{abstract}
In this paper, a general fixed point theorem for cyclic multi-valued mappings satisfying an implicit relation from [19] different from implicit relations used in [13] and [23, generalizing some results from [22, [15, [13, 14], [16], 10] and from other papers, is proved.
\end{abstract}

\section{Introduction}

In 2003, Kirk et al. [11] extended Banach contraction principle to a case of cyclic contractive mappings. In [16], Petric extended most of the fundamental metrical fixed point theorems in literature (Chatterjee, Reich, Hardy-Rogers, Ćirić) to a cyclic contractive mappings. Other new results are obtained in [22], [13], [14], [15], [16], [5], [9], [10]. Several extensions of these results have appeared in literature.

On the other hand, Banach's contraction principle is extended to multivalued mappings by Nadler in [12]. Afterward, an interesting and rich fixed point theory for set-valued mapping was developed in many directions. The theory of multi-valued mappings has applications in optimization problem, control theory, differential and integral theory, economics, informatics and

Received: 18.03.2015. Revised: 02.07.2015.

(2010) Mathematics Subject Classification: 54H25, 47H10.

Key words and phrases: fixed point, multi-valued function, cyclical contraction, implicit relation. 
many branches of analysis. Quite recently, Sintunavarat and Kumam [23] initiated the study of common fixed points for cyclic generalized multi-valued contraction mappings.

The study of fixed points for mappings satisfying implicit relations is initiated in [17], [18], [5], [6], [3]. Quite recently, the method is used in the study of fixed points for mappings satisfying a contractive condition of integral type, in fuzzy metric spaces and intuitionistic metric spaces. With this method, the proofs of some fixed point theorems are more simple. Also, the method allows the study of local and global properties of fixed point structures.

Quite recently, Nashin et al. [13] used a method from [1] and introduced an implicit relation-type-cyclic contractive for mappings in metric spaces for such mappings.

The study of fixed points for multi-valued mappings satisfying an implicit relation is initiated in [19], [20] and other papers.

In this paper, a general fixed point theorem for cyclic multi-valued mappings satisfying an implicit relation from [19] different from implicit relations used in [13] and [23], generalizing some results from [22], [11], [13, [14, [16] and from other papers, is proved.

\section{Preliminaries}

Definition 2.1 ([1], 15]). Let $(X, d)$ be a metric space. Let $p$ be a positive integer, $A_{1}, A_{2}, \ldots, A_{p}$ be nonempty closed subsets of $X, Y=\bigcup_{i=1}^{p} A_{i}$ and $T: Y \rightarrow Y$. Then $Y$ is said to be a cyclical representation of $Y$ with respect to $T$ if

1) $A_{i}, i=1,2, \ldots, p$, are nonempty closed sets,

2) $T\left(A_{1}\right) \subset A_{2}, T\left(A_{2}\right) \subset A_{3}, \ldots, T\left(A_{p-1}\right) \subset A_{p}, T\left(A_{p}\right) \subset A_{1}$.

In [2] is introduced a new class of implicit relations.

Definition 2.2(2]). Let $\mathcal{T}$ be the set of all real continuous function $T: \mathbb{R}_{+}^{6} \rightarrow \mathbb{R}$ where $\mathbb{R}$ is the set of all real numbers and $\mathbb{R}_{+}=[0, \infty)$, satisfying the following conditions:

$\left(T_{1}\right) T\left(t_{1}, \ldots, t_{6}\right)$ is non-increasing in variables $t_{2}, \ldots, t_{6}$,

$\left(T_{2}\right)$ there exists a right continuous function $f: \mathbb{R}_{+} \rightarrow \mathbb{R}, f(0)=0, f(t)<t$ for $t>0$, such that for $u, v>0$, the condition $T(u, v, u, v, 0, u+v) \leq 0$ or $T(u, v, 0,0, v, v) \leq 0$ implies $u \leq f(v)$,

$\left(T_{3}\right) T(u, 0, u, 0,0, u)>0, T(u, u, 0,0, u, u)>0, \forall u>0$. 
Definition 2.3. Let $(X, d)$ be a metric space, $p$ a positive integer, $A_{1}$, $A_{2}, \ldots, A_{p}$ nonempty closed subsets of $X$ and $Y=\bigcup_{i=1}^{p} A_{i}$. An operator $f: Y \rightarrow Y$ is called an implicit relation type cyclic contractive mappings if

1) $Y=\bigcup_{i=1}^{p} A_{i}$ is a cyclic representation of $Y$ with respect to $f$, 2) for every $(x, y) \in A_{i} \times A_{i+1}, i=1,2, \ldots, p$ and $A_{p+1}=A_{1}$,

$$
T(d(f x, f y), d(x, y), d(x, f x), d(y, f y), d(x, f y), d(y, f x)) \leq 0
$$

for some $T \in \mathcal{T}$.

The following theorem is proved in [13].

TheOrem 2.1. Let $(X, d)$ be a complete metric space, $p \in \mathbb{N}, A_{1}, \ldots, A_{p}$, nonempty closed subsets of $X$ and $Y=\bigcup_{i=1}^{p} A_{i}$. Suppose that $f: Y \rightarrow Y$ is an implicit relation type-cyclic contractive mapping for some $T \in \mathcal{T}$. Then $f$ has an unique fixed point. Moreover, the fixed point of $f$ belongs to $\bigcap_{i=1}^{p} A_{i}$.

By this theorem, as particular cases we obtain some results by [11], [14], [15], [16].

Let $(X, d)$ be a metric space. We denote by $C B(X)$ the set of all nonempty bounded closed subsets of $(X, d)$ and by $H$ the Hausdorff-Pompeiu metric on $C B(X)$, i.e.

$$
H(A, B)=\max \left\{\sup _{x \in A}\{d(x, B)\}, \sup _{x \in B}\{d(x, A)\}\right\},
$$

where $A, B \in C B(X)$ and

$$
d(x, A)=\inf _{y \in A}\{d(x, y)\} .
$$

It is well known that $(C B(X), H)$ is a metric space and the completness of $(X, d)$ implies the completness of $(C B(X), H)$.

Let $A, B \in C B(X)$ and $k>1$. In the sequence, the following well known fact will be used [12]: for $a \in A$, there is $b \in B$ such that

$$
d(a, b) \leq k H(A, B) .
$$

Definition 2.4. Let $(X, d)$ be a metric space and $F: X \rightarrow C B(X)$, a multi-valued mappings. A point $x \in X$ is a fixed point of $F$ if $x \in F x$.

In the following we denote by $\operatorname{Fix}(F)$ the set of all fixed points of $F$. 
Definition 2.5. Let $(X, d)$ be a metric space, $p$ be a positive integer, $A_{1}, A_{2}, \ldots, A_{p}$ be nonempty closed subsets of $X, Y=\bigcup_{i=1}^{p} A_{i}$ and $F: Y \rightarrow$ $C B(X)$. Then $Y$ is called a cyclic representation of $Y$ with respect to $F$ if:

1) $A_{i}, i=1,2, \ldots, p$, are nonempty closed sets,

2) $F\left(A_{1}\right) \subset A_{2}, F\left(A_{2}\right) \subset A_{3}, \ldots, F\left(A_{p-1}\right) \subset A_{p}, F\left(A_{p}\right) \subset A_{1}$.

\section{Implicit relations}

Definition 3.1. Let $\mathcal{T}_{6}$ be the family of all real continuous functions $T\left(t_{1}, \ldots, t_{6}\right): \mathbb{R}_{+}^{6} \rightarrow \mathbb{R}$ satisfying the following conditions:

$\left(T_{1}\right): T$ is decreasing in variable $t_{3}, t_{4}, t_{5}$;

$\left(T_{2}\right)$ : there exist $h \in[0,1)$ and $k>1$ such that for all $u, v, t \geq 0, u \leq k t$ and $T(t, v, v, u, u+v, 0) \leq 0$ implies $u \leq h v$.

EXAMPLE 3.1. $T\left(t_{1}, \ldots, t_{6}\right)=t_{1}-a_{1} t_{2}-a_{2} t_{3}-a_{3} t_{4}-a_{4} t_{5}-a_{5} t_{6}$, where $a_{1}, \ldots, a_{5} \geq 0$ and $a_{1}+a_{2}+a_{2}+2 a_{4}<1$.

$\left(T_{1}\right)$ : Obviously.

$\left(T_{2}\right):$ Let $u, v, t \geq 0$,

$$
1<k<\frac{1}{a_{1}+a_{2}+a_{2}+2 a_{4}},
$$

$u \leq k t$ and $T(t, v, v, u, u+v, 0)=t-a_{1} v-a_{2} v-a_{3} v-a_{4}(u+v) \leq 0$. Then $u \leq k t \leq k\left(a_{1} v+a_{2} v+a_{3} u-a_{4}(u+v)\right)$ which implies $u \leq h v$, where

$$
0 \leq h=\frac{k\left(a_{1}+a_{2}+a_{4}\right)}{1-\left(k a_{3}+k a_{4}\right)}<1 .
$$

ExAmple 3.2. $T\left(t_{1}, \ldots, t_{6}\right)=t_{1}-p \max \left\{t_{2}, t_{3}, \ldots, t_{6}\right\}$, where $p \in\left[0, \frac{1}{2}\right)$. $\left(T_{1}\right)$ : Obviously.

$\left(T_{2}\right)$ : Let $u, v, t \geq 0,1<k<\frac{1}{2 p}, u \leq k t$ and $T(t, v, v, u, u+v, 0)=$ $t-p(u+v) \leq 0$. Then $u \leq k t \leq k p(u+v)$, which implies $u \leq h v$, where

$$
0 \leq h=\frac{k p}{1-k p}<1 .
$$

EXAMPle 3.3. $T\left(t_{1}, \ldots, t_{6}\right)=t_{1}-p \max \left\{t_{2}, t_{3}, t_{4}, \frac{t_{5}+t_{6}}{2}\right\}$, where $p \in[0,1)$. $\left(T_{1}\right)$ : Obviously. 
$\left(T_{2}\right):$ Let $u, v, t \geq 0,1<k<\frac{1}{p}, u \leq k t$ and $T(t, v, v, u, u+v, 0)=$ $t-p \max \left\{u, v, \frac{u+v}{2}, 0\right\} \leq 0$. If $u>v$, then $u \leq k p u<u$, a contradiction. Hence $u \leq v$, which implies $u \leq h v$, where $0 \leq h=k p<1$.

EXAmple 3.4. $T\left(t_{1}, \ldots, t_{6}\right)=t_{1}-p \max \left\{t_{2}, \frac{t_{3}+t_{4}}{2}, \frac{t_{5}+t_{6}}{2}\right\}$, where $p \in$ $[0,1)$. The proof is similar to the proof from Example 3.3 .

ExAmple 3.5. $T\left(t_{1}, \ldots, t_{6}\right)=t_{1}^{2}-a t_{2}^{2}-b t_{3} t_{4}-t_{5} t_{6}$, where $a, b \geq 0$ and $0<a+b<1$.

$\left(T_{1}\right)$ : Obviously.

$\left(T_{2}\right)$ : Let $u, v, t \geq 0,1<k<\frac{1}{\sqrt{a+b}}, u \leq k t$ and $T(t, v, v, u, u+v, 0)=$ $t^{2}-a v^{2}-b u v \leq 0$. Then $u^{2} \leq k^{2} t^{2} \leq k^{2}\left(a v^{2}+b u v\right)$. If $u>v$, then $u^{2} \leq$ $k^{2}(a+b) u^{2}<u^{2}$, a contradiction. Hence $u \leq v$, which implies $u \leq h v$, where $0 \leq h=k \sqrt{a+b}<1$.

EXAMPLE 3.6.

$$
T\left(t_{1}, \ldots, t_{6}\right)=t_{1}^{2}+\frac{t_{1}}{1+t_{5}+t_{6}}-\left(a t_{2}^{2}+b t_{3}^{2}-c t_{4}^{2}\right),
$$

where $a, b, c \geq 0$ and $0<a+b+c<1$.

$\left(T_{1}\right)$ : Obviously.

$\left(T_{2}\right):$ Let $u, v, t \geq 0,1<k<\frac{1}{\sqrt{a+b+c}}, u \leq k t$ and $T(t, v, v, u, u+v, 0)=$ $t^{2}+t-a v^{2}-b v^{2}-c u^{2} \leq 0$, which implies $t^{2} \leq a v^{2}+b v^{2}+c u^{2}$. Then $u^{2} \leq k^{2} t^{2} \leq$ $k^{2}\left(a v^{2}+b v^{2}+c u^{2}\right)$, which implies $u \leq h v$, where $0 \leq h=k \sqrt{a+b+c}<1$.

EXAmple 3.7. $T\left(t_{1}, \ldots, t_{6}\right)=t_{1}^{2}-p \max \left\{t_{2}^{2}, t_{3} t_{4}, \frac{1}{2}\left(t_{3} t_{5}+t_{4} t_{6}\right)\right\}$, where $p \in[0,1)$.

$\left(T_{1}\right)$ : Obviously.

$\left(T_{2}\right):$ Let $u, v, t \geq 0,1<k<\frac{1}{\sqrt{p}}, u \leq k t$ and $T(t, v, v, u, u+v, 0)=$ $t^{2}-p \max \left\{v^{2}, u v, \frac{1}{2}[v(u+v)]\right\} \leq 0$. Then $u^{2} \leq k^{2} t^{2} \leq k^{2} p \max \left\{v^{2}, u v\right.$, $\left.\frac{1}{2}\left(u v+v^{2}\right)\right\}$. If $u>v$, then $u^{2} \leq k^{2} t^{2} \leq k^{2} p u^{2}<u^{2}$, a contradiction. Hence $u \leq v$, which implies $u \leq h v$, where $0 \leq h=k \sqrt{p}<1$.

EXAMPle 3.8. $T\left(t_{1}, \ldots, t_{6}\right)=t_{1}^{2}-p \max \left\{t_{2}^{2}, t_{3} t_{5}, t_{4} t_{6}\right\}$, where $p \in\left[0, \frac{1}{2}\right)$. $\left(T_{1}\right)$ : Obviously.

$\left(T_{2}\right)$ : Let $u, v, t \geq 0,1<k<\frac{1}{\sqrt{2 p}}, u \leq k t$ and $T(t, v, v, u, u+v, 0)=$ $t^{2}-p \max \left\{v^{2}, v(u+v)\right\} \leq 0$. Then $u^{2} \leq k^{2} t^{2} \leq k^{2} p \max \left\{v^{2}, v(u+v)\right\}$. If $u>v$, then $u^{2} \leq 2 p k^{2}<u^{2}$, a contradiction. Hence $u \leq v$, which implies $u \leq h v$, where $0 \leq h=k \sqrt{2 p}<1$. 
Definition 3.2. Let $(X, d)$ be a metric space. Let $p$ be a positive integer, $A_{1}, A_{2}, \ldots, A_{p}$ be nonempty closed subsets of $X, Y=\bigcup_{i=1}^{p} A_{i}$. A multi-valued $F: Y \rightarrow C B(X)$ is called an implicit cyclic contractive mapping if:

1) $Y=\bigcup_{i=1}^{p} A_{i}$ is a cyclic representation of $Y$ with respect to $F$,

2) for any $(x, y) \in A_{i} \times A_{i+1}, i=1,2, \ldots, p$ and $A_{p+1}=A_{1}$,

(3.1) $T(H(F x, F y), d(x, y), d(x, F x), d(y, F y), d(x, F y), d(y, F x)) \leq 0$

for some $T \in \mathcal{T}_{6}$.

In this paper we extend Theorem 2.1 for multi-valued mappings.

\section{Main results}

TheOREm 4.1. Let $(X, d)$ be a complete metric space, $p \in \mathbb{N}, A_{1}, \ldots, A_{p}$ be nonempty closed subsets of $X$ and $Y=\bigcup_{i=1}^{p} A_{i}$. If $F: Y \rightarrow C B(X)$ is an implicit cyclic contractive mapping for some $T \in \mathcal{T}_{6}$. Then $F$ has at least a fixed point in $\bigcap_{i=1}^{p} A_{i}$.

Proof. Let $x_{0} \in A_{1}$ and $x_{1} \in T x_{0} \subset A_{2}$. Then, there exists $x_{2} \in T x_{1} \subset$ $A_{3}$ such that

$$
d\left(x_{1}, x_{2}\right) \leq k H\left(T x_{0}, T x_{1}\right) .
$$

Similarly, there exists $x_{3} \in T x_{2} \subset A_{4}$ such that

$$
d\left(x_{2}, x_{3}\right) \leq k H\left(T x_{1}, T x_{2}\right),
$$

and there exists $x_{4} \in T x_{3} \subset A_{5}$ such that

$$
d\left(x_{3}, x_{4}\right) \leq k H\left(T x_{2}, T x_{3}\right),
$$

and there exists $x_{p-2} \in T x_{p-3} \subset A_{p-1}$ and $x_{p-1} \in T x_{p-2} \subset A_{p}$ such that

$$
d\left(x_{p-2}, x_{p-1}\right) \leq k H\left(T x_{p-3}, T x_{p-2}\right) .
$$

Similarly, there exists $x_{p} \in T x_{p-1} \subset A_{p+1}=A_{1}$ such that

$$
d\left(x_{p-1}, x_{p}\right) \leq k H\left(T x_{p-2}, T x_{p-1}\right) .
$$


Hence, using this method, we defined a sequence $\left(x_{n}\right)$ in $X$ which contain the following subsequences

$$
\begin{aligned}
\left\{x_{n p}, n=0,1,2, \ldots\right\} & \subset A_{1}, \\
\left\{x_{n p+1}, n=0,1,2, \ldots\right\} & \subset A_{2}, \\
\vdots & \\
\left\{x_{(n+1) p-1}, n\right. & =0,1,2, \ldots\} \subset A_{p} .
\end{aligned}
$$

If $x=x_{0} \in A_{1}$ and $y=x_{1} \in A_{2}$ we obtain by (3.1) that

$T\left(H\left(T x_{0}, T x_{1}\right), d\left(x_{0}, x_{1}\right), d\left(x_{0}, T x_{0}\right), d\left(x_{1}, T x_{1}\right), d\left(x_{0}, T x_{1}\right), d\left(x_{1}, T x_{0}\right)\right) \leq 0$.

This by $\left(T_{1}\right)$ leads to

$$
T\left(H\left(T x_{0}, T x_{1}\right), d\left(x_{0}, x_{1}\right), d\left(x_{0}, x_{1}\right), d\left(x_{1}, x_{2}\right), d\left(x_{0}, x_{2}\right), 0\right) \leq 0 .
$$

By $\left(T_{1}\right)$ and triangle inequality we have

$$
T\left(H\left(T x_{0}, T x_{1}\right), d\left(x_{0}, x_{1}\right), d\left(x_{0}, x_{1}\right), d\left(x_{1}, x_{2}\right), d\left(x_{0}, x_{1}\right)+d\left(x_{1}, x_{2}\right), 0\right) \leq 0 .
$$

Then by (4.1) and $\left(T_{2}\right)$ we obtain

$$
d\left(x_{1}, x_{2}\right) \leq h d\left(x_{0}, x_{1}\right) .
$$

Similarly, by 4.2 and $\left(T_{2}\right)$ we obtain

$$
d\left(x_{2}, x_{3}\right) \leq h d\left(x_{1}, x_{2}\right) .
$$

Similarly, by 4.5 and $\left(T_{2}\right)$ we obtain

$$
d\left(x_{p-1}, x_{p}\right) \leq h d\left(x_{p-2}, x_{p-1}\right) .
$$

By induction we obtain

$$
d\left(x_{n}, x_{n-1}\right) \leq h d\left(x_{n-1}, x_{n-2}\right) \leq \ldots \leq h^{n} d\left(x_{0}, x_{1}\right), n=1,2, \ldots .
$$

By a routine calculation it follows that $\left(x_{n}\right)$ is a Cauchy sequence in $X$. Since $(X, d)$ is complete it follows that $\left(x_{n}\right)$ is convergent in $X$ to $x^{*}$. Then, also the subsequences $\left(x_{n p+1}\right),\left(x_{n p+2}\right), \ldots,\left(x_{(n+1) p-1}\right)$ converge to $x^{*}$. Since 
$A_{1}, A_{2}, \ldots, A_{p}$ are closed subsets in $X$, then $x^{*} \in A_{i}, i=1,2, \ldots, p$ which implies that $x^{*} \in \bigcap_{i=1}^{p} A_{i}$.

Definition 4.1. Let $(X, d)$ be a metric space, $p \in \mathbb{N}, A_{1}, \ldots, A_{p}$ be nonempty closed subsets of $X, Y=\bigcup_{i=1}^{p} A_{i}$. A multi-valued mapping $F: Y \rightarrow$ $C B(X)$ is called Kannan-type-cyclic contractive mapping if

1) $Y=\bigcup_{i=1}^{p} A_{i}$ is a cyclic representation of $Y$ with respect to $T$,

2) for any $(x, y) \in A_{i} \times A_{i+1}, i=1,2, \ldots, p$ and $A_{p+1}=A_{1}$,

$$
H(F x, F y) \leq a[d(x, T x)+d(y, T y)]
$$

where $0 \leq a<\frac{1}{2}$.

Corollary 4.1. Let $(X, d)$ be a complete metric space, $p \in \mathbb{N}, A_{1}, \ldots, A_{p}$ nonempty closed subsets of $X$ and $Y=\bigcup_{i=1}^{p} A_{i}$.

If $F: Y \rightarrow C B(X)$ is a Kannan-type-cyclic contractive mapping then $F$ have at least a fixed point in $\bigcap_{i=1}^{p} A_{i}$.

Proof. The proof it follows by Theorem 4.1 and Example 3.1 where $a_{1}=$ $a_{5}=a_{6}=0$ and $a_{2}=a_{3}=a$.

REMARK 4.1. This corollary extends the result by [17] for multi-valued mappings.

Definition 4.2. Let $(X, d)$ be a metric space, $p \in \mathbb{N}, A_{1}, \ldots, A_{p}$ nonempty closed subsets of $X$ and $Y=\bigcup_{i=1}^{p} A_{i}$. A multi-valued mapping $F: Y \rightarrow$ $C B(X)$ is called a Chatterjee-type-cyclic contractive mapping if

1) $Y=\bigcup_{i=1}^{p} A_{i}$ is a cyclic representation of $Y$ with respect to $T$,

2) for any $(x, y) \in A_{i} \times A_{i+1}, i=1,2, \ldots, p$ and $A_{p+1}=A_{1}$,

$$
H(F x, F y) \leq a[d(x, T y)+d(y, T x)],
$$

where $0 \leq a<\frac{1}{2}$.

Corollary 4.2. Let $(X, d)$ be a complete metric space, $p \in \mathbb{N}, A_{1}, \ldots, A_{p}$ nonempty closed subsets of $X$ and $Y=\bigcup_{i=1}^{p} A_{i}$.

If $F: Y \rightarrow C B(X)$ is a Chatterjee-type-cyclic contractive mapping then $F$ have at least a fixed point in $\bigcap_{i=1}^{p} A_{i}$.

Proof. The proof it follows by Theorem 4.1 and Example 3.1 where $a_{1}=$ $a_{2}=a_{3}=0$ and $a_{4}=a_{5}=a$. 
Remark 4.2. This corollary extends [16, Theorem 3] for multi-valued mappings.

Definition 4.3. Let $(X, d)$ be a metric space, $p \in \mathbb{N}, A_{1}, \ldots, A_{p}$ nonempty closed subsets of $X$ and $Y=\bigcup_{i=1}^{p} A_{i}$. A multi-valued mapping $F: Y \rightarrow$ $C B(X)$ is called a Reich-type-cyclic contraction if

1) $Y=\bigcup_{i=1}^{p} A_{i}$ is a cyclic representation of $Y$ with respect to $T$,

2) for all $(x, y) \in A_{i} \times A_{i+1}, i=1,2, \ldots, p$ and $A_{p+1}=A_{1}$,

$$
H(F x, F y) \leq a d(x, y)+b d(x, T x)+c d(y, T y),
$$

where $a, b, c \geq 0$ and $a+b+c<1$.

Corollary 4.3. Let $(X, d)$ be a complete metric space, $p \in \mathbb{N}, A_{1}, \ldots, A_{p}$ nonempty closed subsets of $X$ and $Y=\bigcup_{i=1}^{p} A_{i}$.

If $F: Y \rightarrow C B(X)$ is a Reich-type-cyclic contractive mapping then $F$ have at least a fixed point in $\bigcap_{i=1}^{p} A_{i}$.

Proof. The proof it follows by Theorem 4.1 and Example 3.1 where $a_{1}=$ $a, a_{2}=b, a_{2}=c$ and $a_{4}=a_{5}=0$.

REMARK 4.3. This corollary extends [16, Theorem 7] for multi-valued mappings.

Definition 4.4. Let $(X, d)$ be a metric space, $p \in \mathbb{N}, A_{1}, \ldots, A_{p}$ nonempty closed subsets of $X$ and $Y=\bigcup_{i=1}^{p} A_{i}$. A multi-valued mapping $F: Y \rightarrow$ $C B(X)$ is called a Hardy-Roger-type-cyclic contractive mapping if

1) $Y=\bigcup_{i=1}^{p} A_{i}$ is a cyclic representation of $Y$ with respect to $T$,

2) for all $(x, y) \in A_{i} \times A_{i+1}, i=1,2, \ldots, p$ and $A_{p+1}=A_{1}$,

$$
H(F x, F y) \leq a_{1} d(x, y)+a_{2} d(x, T x)+a_{3} d(y, T y)+a_{4} d(x, T y)+a_{5} d(y, T x),
$$

where $a_{1}, \ldots, a_{5} \geq 0$ and $a_{1}+a_{2}+a_{3}+2 a_{4}<1$.

Corollary 4.4. Let $(X, d)$ be a complete metric space, $p \in \mathbb{N}, A_{1}, \ldots, A_{p}$ nonempty closed subsets of $X$ and $Y=\bigcup_{i=1}^{p} A_{i}$.

If $F: Y \rightarrow C B(X)$ is a Hardy-Rogers-type-cyclic contractive mapping, then $F$ have at least a fixed point in $\bigcap_{i=1}^{p} A_{i}$.

Remark 4.4. This corollary extends [16, Theorem 9] for multi-valued mappings. 
REMARK 4.5 .

a) Also, by Theorem 4.1 and Example 3.1 for $a_{2}=a_{3}=a_{4}=0$ we obtain a result which extend the results from [14, Theorem 3.1 (1)].

b) Also, by Theorem 4.1 and Example 3.3 we obtain an result which extend [13. Corollary 3.3] for multi-valued mappings.

Acknowledgements. The author would like to thank the Editor and anonymous reviewers for their constructive comments, which helped to improve the final version of the paper.

\section{References}

[1] Altun I., Turkoglu D., Some fixed point theorems for weakly compatible mappings satisfying an implicit relation, Taiwanese J. Math. 13 (2009), no. 4, 1291-1304.

[2] Altun I., Simsek H., Some fixed point theorems on ordered metric spaces and applications, Fixed Point Theory Appl. 2010, Art. ID 621469, 17 pp.

[3] Aydi H., Jellali M., Karapinar E., Common fixed points for generalized $\alpha$-implicit contractions in partial metric spaces: Consequences and application, RACSAM-Revista de la Real Academia de Ciencias Exactas, Físicas y Naturales. Serie A. Matemáticas. To appear.

[4] Chatterjee S., Fixed point theorems, C.R. Acad. Bulgare Sci. 25 (1972), 727-730.

[5] Gulyaz S., Karapinar E., Coupled fixed point result in partially ordered partial metric spaces through implicit function, Hacet. J. Math. Stat. 42 (2013), no. 4, 347-357.

[6] Gulyaz S., Karapinar E., Yuce I.S., A coupled coincidence point theorem in partially ordered metric spaces with an implicit relation, Fixed Point Theory Appl. 2013, 2013: $38,11 \mathrm{pp}$.

[7] Hardy G.E., Rogers T.D., A generalization of a fixed point of Reich, Can. Math. Bull. 16 (1973), no. 2, 201-206.

[8] Kannan R., Some results on fixed points, Bull. Calcutta Math. Soc. 10 (1968), 71-76.

[9] Karapinar E., Fixed point theory for cyclic weak $\phi$-contraction, Appl. Math. Lett. 24 (2011), no. 6, 822-825 .

[10] Karapinar E., Erhan I.M., Cyclic contractions and fixed point theorems, Filomat 26 (2012), no. 4, 777-782.

[11] Kirk W.A., Srinivasan P.S., Veeramani P., Fixed points for mappings satisfying cyclical contractive conditions, Fixed Point Theory 4 (2003), no. 1, 79-89.

[12] Nadler S.B., Multivalued contraction mappings, Pacific J. Math. 20 (1969), no. 2, 457488.

[13] Nashine H.K., Kadelburg Z., Kumam P., Implicit-relation-type cyclic contractive mappings and applications to integral equations, Abstr. Appl. Anal. 2012, Art. ID 386253, 15 pp.

[14] Păcurar M., Fixed point theory for cyclic Berinde operators, Fixed Point Theory 12 (2011), no. 2, 419-428.

[15] Păcurar M., Rus I.A., Fixed point theory for cyclic $\varphi$-contractions, Nonlinear Anal. 72 (2010), 1181-1187.

[16] Petric M.A., Some results concerning cyclical contractive mappings, Gen. Math. 18 (2010), no. 4, 213-226.

[17] Popa V., Some fixed point theorems for implicit contractive mappings, Stud. Cercet. Ştiinţ., Ser. Mat., Univ. Bacău 7 (1997), 129-133. 
[18] Popa V., Some fixed point theorems for compatible mappings satisfying an implicit relation, Demonstratio Math. 32 (1999), no. 1, 157-163.

[19] Popa V., A general fixed point theorem for weakly commuting multi-valued mappings, Anal. Univ. Dunărea de Jos, Galaţi, Ser. Mat. Fiz. Mec. Teor., Fasc. II 18 (22) (1999), 19-22.

[20] Popa V., A general coincidence theorem for compatible multivalued mappings satisfying an implicit relation, Demonstratio Math. 33 (2000), no. 1, 159-164.

[21] Reich S., Some remarks concerning contraction mappings, Canad. Math. Bull. 14 (1971), 121-124.

[22] Rus I.A., Cyclic representations of fixed points, Ann. Tiberiu Popoviciu, Semin. Funct. Equ. Approx. Convexity 3 (2005), 171-178.

[23] Sintunavarat W., Kumam P., Common fixed point theorem for cyclic generalized multivalued mappings, Appl. Math. Lett. 25 (2012), 1849-1855.

\author{
Vasile Alecsandri" University of BacăU \\ 157 Calea Mărăşeşti \\ BACĂU, 600115 \\ Romania \\ e-mail: vpopa@ub.ro
}

